\title{
RESEARCH
}

Open Access

\section{Sex-specific associations between lipids and cognitive decline in the middle-aged and elderly: a cohort study of Chinese adults}

Lili Liu $^{1+}$, Chen Zhang ${ }^{2+}$, Xiaozhen $\mathrm{Lv}^{3}$, Xuefeng Lai ${ }^{4}, \mathrm{Lu} \mathrm{Xu}^{1}$, Jingnan Feng ${ }^{1}$, Yongfeng Song ${ }^{2^{*}}$ (D), Shengfeng Wang ${ }^{1 *}$ and Siyan Zhan ${ }^{1,5,6^{*}}$

\begin{abstract}
Background: Studies regarding the lipid-cognition relationship have increasingly gained popularity but have generated much mixed results. To date, few studies have focused on the difference between sexes.

Methods: This study included 6792 Chinese adults aged over 45 years (women, 48.56\%; mean age, 57.28 years), who were free of severe conditions known to affect cognitive function at the baseline (2011). Blood concentrations of total cholesterol (TC), high-density lipoprotein cholesterol (HDL-C), low-density lipoprotein cholesterol (LDL-C), and triglycerides (TG) were assessed at baseline, and both continuous and categorical values were used in final analyses. Global cognitive functions were assessed by the word recall test and the mental status test in 2011, 2013, and 2015, respectively. We graded participants into three groups according to the cognitive change slopes: no decline $(\geq 0)$, moderate decline (median to 0 ), and severe decline ( $<$ median). Sex-specific associations between blood lipids and cognitive decline were analyzed using ordinal logistic models, adjusting for sociodemographic information, lifestyle behaviors, and health status.

Results: Higher baseline TC and LDL-C concentrations exhibited no significant association with 5-year cognitive decline in men but were significantly associated with greater 5 -year cognitive decline in women [odds ratio (OR) 1.026, 95\% confidence interval (CI) 1.003, 1.050; OR 1.026, Cl 1.002, 1.051, respectively]. For higher serum HDL-c levels, a significantly protective effect on cognition was observed in men, but a slightly adverse effect was found in women (not significant after Bonferroni correction). TG presented almost no effect on later cognition in either sex.

Conclusion: Different associations between sexes were observed for the lipid-cognition relationship, and maintaining serum cholesterol levels at an appropriate range may have a positive effect on cognitive health.
\end{abstract}

Keywords: Lipids, Cognitive decline, Sex

\footnotetext{
*Correspondence: syf198506@163.com; shengfeng1984@126.com; siyanzhan@bjmu.edu.cn

${ }^{+}$Lili Liu and Chen Zhang contributed equally to this work.

${ }^{2}$ Department of Endocrinology, Shandong Provincial Hospital Affiliated to Shandong First Medical University, 324 Jing 5 road, Huaiyin District, Jinan 250021, China

'Department of Epidemiology and Biostatistics, School of Public Health, Peking University, 38 Xueyuan Road, Haidian District, Beijing 100191, China Full list of author information is available at the end of the article
}

C The Author(s). 2020 Open Access This article is licensed under a Creative Commons Attribution 4.0 International License, which permits use, sharing, adaptation, distribution and reproduction in any medium or format, as long as you give appropriate credit to the original author(s) and the source, provide a link to the Creative Commons licence, and indicate if changes were made. The images or other third party material in this article are included in the article's Creative Commons licence, unless indicated otherwise in a credit line to the material. If material is not included in the article's Creative Commons licence and your intended use is not permitted by statutory regulation or exceeds the permitted use, you will need to obtain permission directly from the copyright holder. To view a copy of this licence, visit http://creativecommons.org/licenses/by/4.0/. The Creative Commons Public Domain Dedication waiver (http://creativecommons.org/publicdomain/zero/1.0/) applies to the data made available in this article, unless otherwise stated in a credit line to the data. 


\section{Background}

With the population aging rapidly, cognitive impairment has become a serious global public health issue. Approximately 20-30\% of Americans and 8\% Chinese aged over 65 years are affected by cognitive impairment, ranging from mild deficits to dementia [1, 2]. Given that no reversible therapy is currently available for cognitive impairment, it is crucial to identify potential modifiable risk factors for rapid cognitive decline and thus employ corresponding strategies for early intervention.

Dyslipidemia is a well-recognized risk factor for atherosclerosis as well as cardio-cerebrovascular diseases. Since atherosclerosis and vascular diseases are important contributing factors to cognitive decline and dementia [3, 4], exploring the relationship of dyslipidemia with cognitive decline has aroused increasing interest in recent years. However, the results have always been conflicting. A few epidemiological studies suggested that high serum total cholesterol (TC) levels predicted subsequently greater cognitive decline or the onset of dementia in general populations [5-8], and lipid lowering agents might have protective effects [9]. However, other studies showed no [10-12] or reverse associations [13-15].

Sex differences have been noted in lipid profiles, vascular physiology, specific cognitive domains, and progression of dementia apart from hormone status [16-19], and the prevalence of dementia in women exhibits a 1.65-fold increase compared to that in men in China [20]. Different compositions between sexes might thus explain the contradictory findings of those longitudinal studies related to the lipid-cognition associations. However, such population-based studies that are stratified by sex are limited and have drawn contradictory conclusions [5, 21, 22]. A prior longitudinal study in Sweden, where baseline serum TC, HDL-c, and TG levels were higher in women than men, reported that higher $\mathrm{TC}$ in men and lower triglycerides or higher HDL-C in women predicted better maintenance of cognitive abilities [21]. However, another prospective study conducted in China reported no sex difference in the lipidcognition associations [5]. Conversely, in the ThreeCity study of French, a hypercholesterolemic pattern in men and a hypolipidemic pattern in women were associated with an increased risk of cognitive decline after a 7-year follow-up [22]. In this study, women also exhibited different baseline serum cholesterol levels compared with men (increased TC, LDL-C, and HDL-c levels, except TG).

In total, gaps in the knowledge of sex-specific associations between lipids and cognition currently exist, especially in the middle-aged population, and the sample sizes of existing cohort studies were relatively small [5, 21, 22].
This study therefore aimed to explore the sex-specific associations of serum lipids with 5-year cognitive decline in a community-based longitudinal study of Chinese elderly individuals derived from the China Health and Retirement Longitudinal Study (CHARLS) based on its large sample, containing middle-aged participants and its thorough and detailed information on the exposure and outcome.

\section{Methods \\ Study population}

CHARLS is a nationally representative cohort study of 17,424 individuals recruited at ages over 45 years from 450 communities in China [23]. The baseline survey was conducted in 2011-2012, and a face-to-face computerassisted personal interviewing was used to collect sociodemographic information, lifestyle behaviors, and health and cognitive status. Among the study participants, 13, 978 individuals $(78.9 \%)$ provided anthropometric and physical performance measures. In this group, blood samples were collected from 11,847 individuals, yielding a response rate of $67 \%$. Two biennial visits were followed in 2013 and 2015 [24], and details of the program were available elsewhere [23].

For this analysis, we excluded CHARLS participants who did not engage in the blood test $(n=5861)$ at baseline and who did not complete cognitive testing at baseline $(n=1924)$. Other exclusion criteria for the original study involved patients suffering from severe diseases or conditions known to affect cognitive function $(n=2187)$ (e.g., depression, malignant tumors, a history of traumatic brain injury, cerebral infarction or cerebrovascular disease, long-term intake of drugs and medication or dietary supplement to improve cognitive function). Consistent with a previous study [5], 660 individuals with a global cognition score of less than or equal to 5 were also excluded due to cognition function being too impaired to be able to complete the cognitive tests or questionnaires (using 5 as the cut-off point to exclude participants with the $5 \%$ lowest cognitive scores [5]). The survey was approved by the Institutional Review Board of Peking University, China (IRB00001052-11015). All subjects provided written informed consent to participate at each study visit.

\section{Lipid measurement}

Serum total cholesterol (TC), low-density lipoprotein cholesterol (LDL-C), high-density lipoprotein cholesterol (HDL-C), and triglycerides (TG) were determined at baseline by an automatic analyzer using enzymatic colorimetric test, and all tests were performed at the Youanmen Center for Clinical Laboratory of Capital Medical University [23]. We considered concentrations of TC, LDL-C, HDL-C, and TG regardless of fasting 
status, given that $92.03 \%$ of participants had been fasting $>8 \mathrm{~h}$ at the time of blood draw. Blind-duplicate coefficients of variation ranged from 0.7 to $1.8 \%$ [25]. We performed a post hoc analysis to see whether higher TC and LDL-C levels were showed in higher HDL-C grades.

\section{Cognitive assessment}

At each study visit, trained study personnel administered the Word Recall Test (WRT) and the Mental Status Test (MST) in a standard order [26, 27]. Similar to the cognitive measurements used in the American Health and Retirement Study (HRS) [23], two composite measures for cognitive functioning were conducted in this study, including the word recall test (WRT) and mental status test (MST): (1) WRT: This test aimed to assess episodic memory by immediate word recall and delayed word recall. Interviewers read a list of ten words only once, and then, the respondents were asked to recall as many of the words as they could in any order (immediate word recall). Then, approximately $4 \mathrm{~min}$ later, they were asked to recall the same list words again (delayed word recall). The word recall score is based on the average of the number of correct answers, ranging from 0 to 10 . Immediate and delayed recall tests were previously demonstrated to have good construct validity and consistency [28]. (2) MST: This test aimed to assess executive function using the TICS-10 (Telephone Interview for Cognitive Status-10) and figure drawing. The TICS-10 is a well-established and valid measure as the Mini-Mental State Examination (MMSE) used to screen cognitively impaired elderly [29] and involves ten questions, including recalling today's date (month, day, year), the day of the week and season of the year, and serial 7 subtraction from 100 (up to five times). This dimension score was calculated based on the number of correct answers, ranging from 0 to 10 . In the figure drawing, the participants were asked to replicate a figure as similarly as possible, and interviewers would score the answer as 1 if the participants successfully completed this task. Those who failed to complete this task received a score of 0 . The MST was performed once for each participant at each visit.

We used the sum of both of the scores of WRT (0 to 10) and MST (0 to 11) as the global cognition score to represent the respondent's comprehensive cognitive status, with scores ranging from 0 to 21 and a higher score indicating better cognitive function [23]. Consistent with previous studies $[22,30]$, slopes of cognitive decline were first calculated by linear regressions directly to quantify the changes of cognition during 2011-2015 (not adjusting for age and education). To promote the clinical interpretability of outcome, three groups of participants were then graded according to the cognitive change slopes: no decline $(\geq 0)$, moderate decline (median to 0 ), and severe decline ( $<$ median).

\section{Covariate assessment}

Similar to previous studies $[5,6,31]$, potential confounders included age (years), education (illiterate/primary school/middle school and above), marriage (married/divorced/single), residence (urban/rural), leisure time social activity (active/inactive/none), health insurance status (yes/no, as a proxy for socioeconomic status and access to health care), alcohol use (current/ former/never), smoking status (current/former/never), hypertension (yes/no), diabetes (yes/no), and lipidlowering medication use (yes/no). Body mass index (BMI) was calculated as body weight $(\mathrm{kg})$ divided by the square of height $\left(\mathrm{m}^{2}\right)$. Diabetes was defined as a selfreported physician diagnosis of diabetes, $\geq 126 \mathrm{mg} / \mathrm{dL}$ fasting glucose, $\geq 200 \mathrm{mg} / \mathrm{dL}$ non-fasting glucose, or use of diabetes medications [32]. Hypertension was determined as self-reported physician diagnosis of hypertension, measured systolic blood pressure $\geq 140 \mathrm{mmHg}$, measured diastolic blood pressure $\geq 90 \mathrm{mmHg}$, or use of antihypertensive medications. Additional variables used in sensitivity analyses included fasting status at baseline blood draw ( $>8 \mathrm{~h}$, yes/no). After the selection of covariates, we finally adjusted all these potential confounders in the main analyses. However, in the subgroup analyses of age or education, we did not further correct the age or education.

\section{Statistical analyses}

The Student $t$ test and the chi-squared test for categorical variables were used to identify basic differences between sexes. Separate ordinal logistic regression models were used to consider whether baseline lipid levels were associated with later cognitive decline in males and females. Lipid profiles were first put in models as continuous variables per $10 \mathrm{mg} / \mathrm{dl}$. Categorical lipid variables, defined by the Third Report of the Expert Panel on Detection, Evaluation, and Treatment of High Cholesterol in Adults (ATP-III) [33], were analyzed in the same models to promote the clinical interpretability. According to a prior study [34], a minor elevation of cholesterol levels, even in a normal range, was associated with later endothelial dysfunction, which thus might increase the risk of vascular disease caused by elevated cholesterol. Similarly, the fluctuation of lipids within the normal range might also influence the later cognition, and thus, we finally reported both continuous and categorical results due to the clinical significance and the comprehensiveness of results [31]. Furthermore, given that the cognitive decline rate of the elderly group was more rapid than that of middle-aged people $[5,35]$, we also conducted subgroup analyses to determine 
whether there was support for effect measure modification of age. Consistent with previous studies [36, 37], we chose a cut-off of 60 years for these subgroup analyses. To make our results nationally representative, a sample weight, which was calculated using an inverse probability method, was used under the correction for household and individual non-response as well as non-participation in the blood collection [23]. All the multivariable analyses in our study were weighted.

For our primary analyses, we used a complete case approach to address missing data. In the sensitivity analysis, we examined associations after (1) imputing those with a global cognition score of less than or equal to 5 only; (2) additionally imputing those with severe diseases or conditions known to affect cognitive function; (3) not adjusting for obesity, diabetes, and hypertension; (4) excluding participants with obesity, diabetes, or hypertension; and (5) not adjusting for marital and health insurance status. Given that fasting status can impact lipid values, we repeated our primary analyses after restricting them to individuals who were fasting $>8 \mathrm{~h}$ before blood draw.

Stata 15.0 (StataCorp LP) was used for all analyses, and formal hypothesis testing was 2 -sided with a significant level of 0.05 . Throughout the analysis, we report $95 \%$ confidence intervals and a $p$ value of $<0.05$ was considered to be statistically significant. We used the Bonferroni test for multiple corrections.

\section{Results}

\section{Subjects}

A total of 6792 individuals were included in the analysis, including 3494 men and 3298 women, with a mean (SD) age of $59.26(8.95)$ years for men and 57.28 (8.71) years for women $(p<0.001)$. At baseline, women were more frequently divorced or single and with more living in town $(p<0.001)$. The overall mean concentrations of TC, LDL-C, HDL-C, and TG were 192.57 (38.19) mg/dl, $116.57(34.58) \mathrm{mg} / \mathrm{dl}, 50.69$ (14.71) $\mathrm{mg} / \mathrm{dl}$, and 128.79 (83.87) $\mathrm{mg} / \mathrm{dl}$, respectively, and all indices were significantly higher in women except HDL-C. According to the ATP-III, the abnormal rate of these four indices were $38.87 \%, \quad 11.88 \%, 24.60 \%$, and $26.30 \%$, respectively. Clearly, the abnormal rates of the four lipids indices in women were significantly increased compared with those in men (Table 1). Moreover, higher TC and LDL-C levels were noted in the high HDL-C grade (Supplementary Table 1).

\section{Cognitive decline}

The medians of cognitive change slopes used to divide participants were -0.75 . -0.75 and -0.38 for the three cognitive scores, respectively. At baseline, men had higher scores of global cognition [12.31 (3.07) versus
11.34 (3.38), $P<0.001]$ and mental status [8.51 (2.30) versus $7.45(2.62), P<0.001]$ compared with women. However, for episodic memory, women scored better than men [3.89 (1.63) versus 3.80 (1.57), $P=0.018]$. In view of the cognitive decline during 2011-2015, no significant differences were noted between men and women among groups of no decline, moderate decline and severe decline. Furthermore, the proportion of decline (moderate and severe) on three cognitive indices in men was comparable to that in women (Table 2).

\section{Lipids and cognitive decline}

Overall, after multivariate adjustment, higher baseline TC or LDL-C level was significantly associated with a greater 5-year decline in global cognition [OR 1.026, 95\% CI 1.003, 1.050; OR 1.026, 95\% CI 1.002, 1.051] in women, whereas no significant association was observed in men. Furthermore, significant associations were also found between higher baseline TC or LDL-C level and faster 5-year decline on the mental status [OR 1.030, 95\% CI 1.007, 1.054; OR 1.034, 95\% CI 1.010, 1.059] in women, and similarly, no significant association was found in men. In general, no significant association was observed between HDL-C and TG and 5-year cognitive decline. However, we noted that higher HDL-C exhibited a marginally significant association with slower 5-year decline in mental status in men (OR $0.948,95 \%$ CI $0.900,1.000, P=0.051)$ and demonstrated an adverse effect in women (OR 1.044, 95\%CI $0.981,1.110, P=0.173)$. No significant association was found overall between lipids and episodic memory both in men and women (Table 3).

Before Bonferroni correction, analyses compared with lipid categories indicated a significant association between elevated TC and greater 5-year decline of mental status in women. The elevation of LDL-C was associated with greater 5 -year decline on the global cognition and the mental status in women, both showing a lineardose response pattern. No significant association was found between lipid categories of baseline TC and LDL$\mathrm{C}$ levels and the subsequent 5-year cognitive decline in men. Elevated HDL-C was associated with slower 5-year cognitive decline in men on global cognition and the mental status but it showed an adverse effect on global cognition in women. However, after Bonferroni correction, only the association between LDL-C and mental status remained. In total, TG did not exhibit a significant association with any of the three cognitive indices regardless of Bonferroni correction (Table 4).

\section{Sensitivity and subgroup analyses}

Results of our sensitivity analyses were substantially consistent with primary findings, including restricting participants to those with fasting $>8 \mathrm{~h}$; imputing those 
Table 1 Descriptive statistics for eligible individuals at baseline

\begin{tabular}{|c|c|c|c|c|}
\hline Characteristics & Overall, $N=6792$ & Male, $n=3494$ & Female, $n=3298$ & $P$ value \\
\hline Age, mean (SD) & $58.30(8.89)$ & $59.26(8.95)$ & $57.28(8.71)$ & $<0.001$ \\
\hline In married, $n(\%)$ & $6137(90.36)$ & $3246(92.90)$ & $2891(87.66)$ & $<0.001$ \\
\hline Urban, $n(\%)$ & $2740(40.34)$ & $1327(37.98)$ & $1413(42.84)$ & $<0.001$ \\
\hline \multicolumn{5}{|l|}{ Education, $n(\%)$} \\
\hline Illiterate & $1364(20.08)$ & $313(8.96)$ & $1051(31.87)$ & \multirow[t]{3}{*}{$<0.001$} \\
\hline Primary school & $2887(42.51)$ & $1608(46.02)$ & $1279(38.78)$ & \\
\hline Middle school and above & $2541(37.41)$ & $1573(45.02)$ & $968(29.35)$ & \\
\hline \multicolumn{5}{|l|}{ Smoking, n (\%) } \\
\hline Never & $3915(57.65)$ & $874(25.01)$ & $3041(92.24)$ & \multirow[t]{3}{*}{$<0.001$} \\
\hline Former & $1175(17.30)$ & $1035(29.63)$ & $140(4.24)$ & \\
\hline Current & $1701(25.05)$ & $1585(45.36)$ & $116(3.52)$ & \\
\hline \multicolumn{5}{|l|}{ Alcohol use, $n(\%)$} \\
\hline Never & $4026(59.28)$ & $1194(34.17)$ & $2832(85.87)$ & \multirow[t]{3}{*}{$<0.001$} \\
\hline Former & $399(5.87)$ & $329(9.42)$ & $70(2.12)$ & \\
\hline Current & $2367(34.85)$ & $1971(56.41)$ & $396(12.01)$ & \\
\hline Lipid-lowering medication using, $n$ (\%) & $161(1.71)$ & $57(1.69)$ & $59(1.73)$ & 0.900 \\
\hline Health insurance, $n(\%)^{\text {a }}$ & $6438(94.96)$ & $3329(95.39)$ & $3109(94.50)$ & 0.095 \\
\hline \multicolumn{5}{|l|}{ Socially active, $n(\%)$} \\
\hline None & $3925(57.79)$ & $2049(58.64)$ & $1876(56.88)$ & \multirow[t]{3}{*}{0.314} \\
\hline Inactive & $1893(27.87)$ & $949(27.16)$ & $944(28.63)$ & \\
\hline Active & $974(14.34)$ & $496(14.20)$ & $478(14.49)$ & \\
\hline \multicolumn{5}{|l|}{ Body mass index $\left(\mathrm{kg} / \mathrm{m}^{2}\right), n(\%)^{b}$} \\
\hline$<18.5$ & $345(5.21)$ & $194(5.71)$ & $151(4.69)$ & \multirow[t]{4}{*}{$<0.001$} \\
\hline $18.5 \sim 23.9$ & $3378(51.06)$ & $1950(57.37)$ & $1428(44.39)$ & \\
\hline $24.0 \sim 27.9$ & $2080(31.44)$ & $952(28.01)$ & $1128(35.06)$ & \\
\hline$>28.0$ & $813(12.29)$ & $303(8.91)$ & $510(15.86)$ & \\
\hline Diabetes, $n(\%)$ & $931(13.72)$ & $478(13.69)$ & $453(13.75)$ & 0.947 \\
\hline Hypertension, $n$ (\%) & $2717(40.00)$ & $1355(38.78)$ & $1362(41.30)$ & 0.034 \\
\hline Total cholesterol (mg/dl), mean (SD) & $192.57(38.19)$ & $188.25(38.31)$ & $197.17(37.52)$ & $<0.001$ \\
\hline$<200, n(\%)$ & $4081(61.13)$ & $2258(65.62)$ & $1823(56.35)$ & \multirow[t]{3}{*}{$<0.001$} \\
\hline 200 239, n (\%) & $1891(28.33)$ & $882(25.63)$ & 1009 (31.19) & \\
\hline $240+, n(\%)$ & $704(10.54)$ & $301(8.75)$ & $403(12.46)$ & \\
\hline LDL-c (mg/dl), mean (SD) & $116.57(34.58)$ & $113.04(34.38)$ & $120.31(34.39)$ & $<0.001$ \\
\hline$<100, n(\%)$ & $2152(32.26)$ & $1242(36.14)$ & $910(28.15)$ & \multirow[t]{5}{*}{$<0.001$} \\
\hline $100 \sim 129, n(\%)$ & $2392(35.86)$ & $1221(35.53)$ & $1171(36.22)$ & \\
\hline 130 159, n (\%) & $1448(21.72)$ & $695(20.22)$ & $753(23.29)$ & \\
\hline $160 \sim 189, n(\%)$ & $501(7.51)$ & $195(5.67)$ & $306(9.46)$ & \\
\hline $190+, n(\%)$ & $177(2.65)$ & $84(2.44)$ & $93(2.88)$ & \\
\hline $\mathrm{HDL}-\mathrm{c}(\mathrm{mg} / \mathrm{dl})$, mean $(\mathrm{SD})$ & $50.69(14.71)$ & $50.35(15.56)$ & $51.04(13.73)$ & 0.054 \\
\hline$<40, n(\%)$ & $1643(24.60)$ & $948(27.54)$ & $695(21.48)$ & \multirow[t]{3}{*}{$<0.001$} \\
\hline $40 \sim 59, n(\%)$ & 3505 (52.48) & $1713(49.75)$ & $1792(55.38)$ & \\
\hline $60+, n(\%)$ & $1531(22.92)$ & $782(22.71)$ & $749(23.14)$ & \\
\hline
\end{tabular}


Table 1 Descriptive statistics for eligible individuals at baseline (Continued)

\begin{tabular}{|c|c|c|c|c|}
\hline Characteristics & Overall, $N=6792$ & Male, $n=3494$ & Female, $n=3298$ & $P$ value \\
\hline Triglycerides (mg/dl), mean (SD) & $128.79(83.87)$ & $124.33(83.88)$ & $133.52(83.61)$ & $<0.001$ \\
\hline$<150, n(\%)$ & $4908(73.70)$ & $2605(75.93)$ & $2303(71.34)$ & $<0.001$ \\
\hline $150 \sim 199, n(\%)$ & $842(12.65)$ & $382(11.13)$ & $460(14.25)$ & \\
\hline $200+, n(\%)$ & $909(13.65)$ & $444(12.94)$ & $465(14.41)$ & \\
\hline
\end{tabular}

LDL-c low-density lipoprotein cholesterol, $H D L-c$ high-density lipoprotein cholesterol

a 12 missing

b 176 missing

with a low global cognition score or those with severe conditions; not adjusting for obesity, diabetes, hypertension, marital status and health insurance; and excluding participants with obesity, diabetes, or hypertension (Table 5, Supplementary Table 3).

In the subgroup analysis of age, after Bonferroni correction, elevated TC was significantly associated with greater 5-year decline of global cognition among female individuals $<60$ years. Elevated LDL-C was significantly associated with greater 5 -year decline of global cognition and mental status, and higher HDL-C was associated with faster 5-year decline of episodic memory (Fig. 1). However, no other significant associations were found among male individuals $<60$ years and all participants $\geq$ 60 years (Fig. 2).

\section{Discussion}

In this study, different impacts of the baseline lipid profile on later cognitive decline were observed between sexes over a 5-year period. We found that higher serum

Table 2 Cognitive descriptions of eligible individuals

\begin{tabular}{|c|c|c|c|c|}
\hline & Overall, $N=6792$ & Male, $n=3494$ & Female, $n=3298$ & $P$ value \\
\hline \multicolumn{5}{|c|}{ Cognition at baseline, mean (SD) } \\
\hline Global cognition & $11.84(3.26)$ & $12.31(3.07)$ & $11.34(3.38)$ & $<0.001$ \\
\hline Mental status & $7.99(2.52)$ & $8.51(2.30)$ & $7.45(2.62)$ & $<0.001$ \\
\hline Episodic memory & $3.85(1.60)$ & $3.80(1.57)$ & $3.89(1.63)$ & 0.018 \\
\hline \multicolumn{5}{|c|}{ Cognition at 2015, mean (SD) } \\
\hline Global cognition & $10.96(3.93)$ & $11.51(3.60)$ & $10.38(4.16)$ & $<0.001$ \\
\hline Mental status & $7.39(2.91)$ & $7.96(2.65)$ & $6.79(3.04)$ & $<0.001$ \\
\hline Episodic memory & $3.47(1.79)$ & $3.44(1.74)$ & $3.50(1.83)$ & 0.226 \\
\hline \multicolumn{5}{|c|}{ Absolute cognitive decline during 2011-2015, mean (SD) } \\
\hline Global cognition & $0.88(3.44)$ & $0.88(3.38)$ & $0.88(3.50)$ & 0.969 \\
\hline Mental status & $0.58(2.71)$ & $0.59(2.67)$ & $0.57(2.76)$ & 0.781 \\
\hline Episodic memory & $0.37(1.86)$ & $0.37(1.83)$ & $0.37(1.89)$ & 0.982 \\
\hline \multicolumn{5}{|c|}{ Groups of global cognition change, $n(\%)^{\text {a }}$} \\
\hline No decline & $3119(47.55)$ & $1612(47.87)$ & $1507(47.20)$ & 0.859 \\
\hline Moderate decline & $1791(27.30)$ & $914(27.15)$ & $877(27.46)$ & \\
\hline Severe decline & $1650(25.15)$ & $841(24.98)$ & $809(25.34)$ & \\
\hline \multicolumn{5}{|c|}{ Groups of mental status change, $n(\%)^{b}$} \\
\hline No decline & $3637(54.67)$ & $1898(55.37)$ & $1739(53.93)$ & 0.499 \\
\hline Moderate decline & $1531(23.02)$ & $779(22.72)$ & $752(23.33)$ & \\
\hline Severe decline & $1484(22.31)$ & $751(21.91)$ & $733(22.74)$ & \\
\hline \multicolumn{5}{|c|}{ Groups of episodic memory change, $n(\%)^{c}$} \\
\hline No decline & $3442(51.74)$ & $1769(51.60)$ & $1673(51.89)$ & 0.884 \\
\hline Moderate decline & $1683(25.30)$ & $876(25.55)$ & 807 (25.03) & \\
\hline Severe decline & 1527 (22.96) & 783 (22.85) & 744 (23.08) & \\
\hline
\end{tabular}

${ }^{a} 232$ missing, no decline (slope of change $\geq 0$ ), moderate decline (slope of change: median to 0 ) and severe decline (slope of change $<$ median) b 140 missing, no decline (slope of change $\geq 0$ ), moderate decline (slope of change: median to 0 ) and severe decline (slope of change < median)

c 140 missing, no decline (slope of change $\geq 0$ ), moderate decline (slope of change: median to 0 ) and severe decline (slope of change < median) 
Table 3 Adjusted odds ratio in 5-year cognitive decline per $10 \mathrm{mg} / \mathrm{dL}$ lipids at baseline, OR (95\%Cl)

\begin{tabular}{|c|c|c|c|c|c|c|}
\hline & \multicolumn{2}{|l|}{ Global cognition $^{\dagger}$} & \multicolumn{2}{|l|}{ Mental status $^{\dagger}$} & \multicolumn{2}{|l|}{ Episodic memory $^{\dagger}$} \\
\hline & Male & Female & Male & Female & Male & Female \\
\hline Total cholesterol & $1.000(0.979,1.021)$ & $1.026(1.003,1.050) *$ & $0.997(0.976,1.018)$ & $1.030(1.007,1.054) * *$ & $1.000(0.978,1.022)$ & $1.003(0.981,1.026)$ \\
\hline LDL-C & $0.995(0.972,1.018)$ & $1.026(1.002,1.051) *$ & $0.994(0.971,1.017)$ & $1.034(1.010,1.059) * *$ & $1.001(0.978,1.025)$ & $0.997(0.973,1.022)$ \\
\hline |l-c & $0.956(0.907,1.007)$ & $1.059(0.995,1.128)$ & $0.948(0.900,1.000)$ & $1.044(0.981,1.110)$ & $0.970(0.921,1.022)$ & $1.059(0.997,1.125)$ \\
\hline Triglycerides & $1.005(0.995,1.016)$ & $0.998(0.989,1.007)$ & $1.004(0.994,1.015)$ & $0.999(0.990,1.009)$ & $1.001(0.991,1.011)$ & $0.999(0.988,1.011)$ \\
\hline
\end{tabular}

$O R$ odds ratio, $C l$ confidence interval, $L D L-c$ low-density lipoprotein cholesterol, $H D L-c$ high-density lipoprotein cholesterol ${ }^{*} P<0.05$

${ }^{*} P<0.01$

${ }^{\dagger}$ Adjusted for baseline age, education, marital status, registered residence, body mass index, alcohol use, smoking status, diabetes, hypertension, social activity, health insurance status, and lipid-lowering medication use

TC and LDL-C levels were significantly associated with greater cognitive decline in global cognition and mental status in women. However, these associations were not found in men. Furthermore, a significant protective effect was also observed for higher serum HDL-C levels on cognition in men, but a slightly adverse effect was found in women. TG exhibited almost no effect on cognition in both sexes.

In our study, higher TC and LDL-C levels were significantly associated with greater 5-year cognitive decline in women. This finding was levels consistent with that of a longitudinal study focused on 1037 postmenopausal women [38], and similar results were also shown in another cohort study that included 1159 Chinese participants over 60 years of age [5]. Actually, there are two potential explanations for this finding: (1) the atherosclerosis caused by higher TC and LDL-c levels, subsequently induced cerebral hypoperfusion, which played a significant role in the acceleration of cognitive decline [39, 40], and (2) higher TC levels might modulate enzymatic processing of the amyloid precursor protein and accelerate the deposition of $\beta$-amyloid in brain, thus increasing the cognitive decline [41, 42]. However, different findings were noted compared to our study. The

Table 4 Adjusted odds ratio in 5-year cognitive change across ATP-III lipid categories at baseline, OR (95\%Cl)

\begin{tabular}{|c|c|c|c|c|c|c|}
\hline & \multicolumn{2}{|l|}{ Global cognition $^{\dagger}$} & \multicolumn{2}{|l|}{ Mental status $^{\dagger}$} & \multicolumn{2}{|l|}{ Episodic memory $^{\dagger}$} \\
\hline & Male & Female & Male & Female & Male & Female \\
\hline \multicolumn{7}{|c|}{ Total cholesterol (mg/dl) } \\
\hline$<200$ & ref & ref & ref & ref & ref & ref \\
\hline $200 \sim 239$ & $1.011(0.848,1.206)$ & $1.010(0.833,1.226)$ & $1.179(0.983,1.414)$ & $1.175(0.972,1.421)$ & $1.035(0.869,1.233)$ & $0.914(0.752,1.111)$ \\
\hline $240+$ & $1.051(0.807,1.369)$ & $1.242(0.965,1.600)$ & $0.889(0.681,1.162)$ & $1.295(1.002,1.675)^{b}$ & $1.040(0.780,1.387)$ & $1.014(0.791,1.300)$ \\
\hline \multicolumn{7}{|c|}{ LDL-c (mg/dl) } \\
\hline$<100$ & ref & ref & ref & Ref & ref & ref \\
\hline $100 \sim 129$ & $0.979(0.813,1.178)$ & $1.308(1.051,1.627)^{b}$ & $0.996(0.827,1.199)$ & $1.302(1.047,1.618)^{b}$ & $1.004(0.835,1.207)$ & $1.207(0.973,1.496)$ \\
\hline 130 159 & $0.918(0.727,1.160)$ & $1.291(1.017,1.639)^{b}$ & $0.984(0.776,1.248)$ & $1.357(1.071,1.720)^{\mathrm{a}}$ & $0.943(0.748,1.189)$ & $1.058(0.828,1.351)$ \\
\hline $160 \sim 189$ & $0.976(0.696,1.367)$ & $1.377(1.002,1.891)^{b}$ & $0.873(0.611,1.249)$ & $1.491(1.076,2.066)^{b}$ & $1.134(0.790,1.629)$ & $1.120(0.819,1.531)$ \\
\hline $190+$ & $0.998(0.644,1.546)$ & $1.424(0.933,2.172)$ & $0.807(0.505,1.289)$ & $1.283(0.838,1.967)$ & $1.283(0.802,2.054)$ & $0.903(0.576,1.415)$ \\
\hline \multicolumn{7}{|c|}{ HDL-c (mg/dl) } \\
\hline$<40$ & ref & ref & ref & ref & ref & ref \\
\hline $40 \sim 59$ & $0.822(0.679,0.994)$ a & $1.159(0.928,1.447)$ & $0.808(0.665,0.980)^{b}$ & $1.161(0.933,1.443)$ & $0.861(0.711,1.042)$ & $1.087(0.873,1.354)$ \\
\hline $60+$ & $0.794(0.629,1.001)$ & $1.294(1.002,1.671)^{b}$ & $0.769(0.614,0.964)^{b}$ & $1.246(0.967,1.606)$ & $0.898(0.711,1.134)$ & $1.245(0.966,1.605)$ \\
\hline \multicolumn{7}{|c|}{ Triglycerides (mg/dl) } \\
\hline$<150$ & ref & ref & ref & ref & ref & ref \\
\hline $150 \sim 199$ & $0.981(0.766,1.257)$ & $1.015(0.795,1.296)$ & $1.008(0.781,1.302)$ & $1.184(0.938,1.494)$ & $0.870(0.675,1.120)$ & $0.800(0.630,1.015)$ \\
\hline $200+$ & $1.088(0.863,1.371)$ & $0.836(0.663,1.054)$ & $1.097(0.864,1.394)$ & $0.955(0.760,1.200)$ & $1.043(0.819,1.329)$ & $0.804(0.628,1.031)$ \\
\hline
\end{tabular}

$O R$ odds ratio, $C l$ confidence interval, $L D L-c$ low-density lipoprotein cholesterol, $H D L-c$ high-density lipoprotein cholesterol

${ }^{\dagger}$ Adjusted for baseline age, education, marital status, registered residence, body mass index, alcohol use, smoking status, diabetes, hypertension, social activity, health insurance status, and lipid-lowering medication use

${ }^{a} P<0.05$ after the Bonferroni correction

${ }^{\mathrm{b}} \mathrm{P}<0.05$ before the Bonferroni correction 
Table 5 Adjusted odds ratio in 5-year cognitive change per $10 \mathrm{mg} / \mathrm{dL}$ lipids at baseline across all sensitivity analyses, OR (95\%Cl)

\begin{tabular}{|c|c|c|c|c|c|c|}
\hline & \multicolumn{2}{|c|}{ Global cognition $^{+}$} & \multicolumn{2}{|l|}{ Mental status $^{\dagger}$} & \multicolumn{2}{|c|}{ Episodic memory $^{\dagger}$} \\
\hline & Male & Female & Male & Female & Male & Female \\
\hline \multicolumn{7}{|c|}{ Sensitivity: Restrict to those fasting $>8 \mathrm{~h}$} \\
\hline Total cholesterol & $1.00(0.98,1.02)$ & $1.02(1.00,1.05)$ & $1.00(0.98,1.02)$ & $1.03(1.00,1.05) *$ & $1.00(0.98,1.02)$ & $1.00(0.98,1.02)$ \\
\hline LDL-c & $1.00(0.98,1.02)$ & $1.03(1.00,1.05) *$ & $1.00(0.98,1.02)$ & $1.04(1.01,1.06)^{* *}$ & $1.00(0.98,1.03)$ & $1.00(0.97,1.02)$ \\
\hline HDL-c & $0.95(0.90,1.00)$ & $1.07(1.00,1.14)$ & $0.94(0.89,0.99) *$ & $1.04(0.98,1.11)$ & $0.97(0.92,1.02)$ & $1.06(0.99,1.13)$ \\
\hline Triglycerides & $1.01(0.99,1.02)$ & $0.99(0.98,1.00)$ & $1.00(0.99,1.02)$ & $1.00(0.99,1.01)$ & $1.00(0.99,1.01)$ & $1.00(0.99,1.01)$ \\
\hline \multicolumn{7}{|c|}{ Sensitivity: Impute those with a global cognition score of less than or equal to 5} \\
\hline Total cholesterol & $1.00(0.98,1.02)$ & $1.02(1.00,1.04) *$ & $1.00(0.98,1.02)$ & $1.03(1.01,1.05) *$ & $1.00(0.98,1.02)$ & $1.00(0.98,1.03)$ \\
\hline LDL-c & $1.00(0.97,1.02)$ & $1.02(1.00,1.04)$ & $0.99(0.97,1.02)$ & $1.03(1.01,1.06)^{* *}$ & $1.00(0.98,1.03)$ & $1.00(0.97,1.02)$ \\
\hline $\mathrm{HDL}-\mathrm{C}$ & $0.96(0.91,1.01)$ & $1.04(0.98,1.10)$ & $0.95(0.90,1.00)^{*}$ & $1.04(0.98,1.11)$ & $0.97(0.92,1.02)$ & $1.06(1.00,1.13)$ \\
\hline Triglycerides & $1.00(0.99,1.01)$ & $1.00(0.99,1.01)$ & $1.00(0.99,1.01)$ & $1.00(0.99,1.01)$ & $1.00(0.99,1.01)$ & $1.00(0.99,1.01)$ \\
\hline \multicolumn{7}{|c|}{ Sensitivity: Impute those with severe diseases or conditions known to affect cognitive function } \\
\hline Total cholesterol & $1.00(0.98,1.02)$ & $1.02(1.00,1.03)$ & $1.00(0.98,1.02)$ & $1.03(1.01,1.05)^{*}$ & $1.00(0.98,1.02)$ & $1.00(0.98,1.03)$ \\
\hline LDL-C & $1.00(0.98,1.02)$ & $1.01(0.99,1.03)$ & $0.99(0.97,1.02)$ & $1.03(1.01,1.06)^{* *}$ & $1.00(0.98,1.03)$ & $1.00(0.97,1.02)$ \\
\hline HDL-c & $0.96(0.91,1.00)$ & $1.02(0.97,1.07)$ & $0.95(0.90,1.00) *$ & $1.04,0.98,1.11)$ & $0.97(0.92,1.02)$ & $1.06(1.00,1.13)$ \\
\hline Triglycerides & $1.00(0.99,1.01)$ & $1.00(0.99,1.01)$ & $1.00(0.99,1.01)$ & $1.00(0.99,1.01)$ & $1.00(0.99,1.01)$ & $1.00(0.99,1.01)$ \\
\hline
\end{tabular}

$O R$ odds ratio, $C I$ confidence interval, $L D L-c$ low-density lipoprotein cholesterol, $H D L-c$ high-density lipoprotein cholesterol

${ }^{*} P<0.05$

${ }^{* *} P<0.01$

${ }^{+}$Adjusted for baseline age, education, marital status, registered residence, body mass index, alcohol use, smoking status, diabetes, hypertension, social activity, health insurance status, and lipid-lowering medication use

WHAS II study that followed 436 community-dwelling women aged $70-79$ years for 9 years indicated that no relationship existed between baseline lipids and cognitive decline [43]. However, the participants in this study who had survived to old age would suffer from more complications and were more selective; thus, these women were not susceptible to the adverse effects of higher lipid levels [43]. This result was consistent with that in our subgroup analyses of age, namely, no significant lipidcognition association was found in individuals $\geq 60$ years. Furthermore, it should be noted that its sample size was relatively small; thus, the statistical power might not be sufficient to detect a moderate effect [44]. A similar phenomenon also occurred, when using $\mathrm{TC}$ as a categorical variable instead of a continuous variable to explore TC-cognition associations in women [44].

In fact, these effects of TC and LDL-C were exclusively found in women rather than in men, revealing a distinct sex difference for the effect of cholesterol, which also existed in previous studies [21, 22]. Three possible mechanisms were considered. First, the vascular physiology naturally differs between sexes. Compared to men, women have smaller arterial sizes and increased vascular remodeling under atherosclerosis [19], which may cause more microvascular damages and hypoperfusion in the brain [45]. White matter lesions occur more commonly in women compared with men, which may further support this point of view [46]. Second, different hormone levels changes between sexes may be another possible reason. Gonadal hormones (e.g., estradiol and testosterone) serve neuroprotective effects across the human lifespan [47]. Generally, women will experience a dramatic loss of estradiol following menopause, whereas testosterone gradually decline in men as age increases [48]. Thus, given the attenuated neuroprotective effect of estrogen, women seemed to be more vulnerable to the adverse effects of high lipid levels on cognition. Third, as the cerebral brain volume is generally greater in men than in women $(\sim 10 \%)$ [49], the concept of brain reserve was proposed by Katzman et al. [50], suggesting that subjects with larger brain reserve have greater capacity to withstand more pathological conditions. Therefore, compared to women, men were expected to have stronger abilities to fight against the adverse pathological progression of hyperlipidemia. However, an opposite viewpoint was also proposed by Sundermann et al. that women would have a greater cognitive reserve than men due to their innate advantages of verbal memory [51]. However, given that a precise mechanism has not been reported to date, more studies are needed to explore the potential mechanisms for this sex difference in lipid-cognition associations.

Regarding serum HDL-C, no significant effect on cognitive decline was found in men or women after Bonferroni correction, which is consistent with a few prior studies $[5,6,8]$. However, of note, before correction, a protective effect of HDL-C on global cognition and mental status was observed in men, which is consistent with the results of Japanese cohort study [52]. HDL-C can transport excess cholesterol in peripheral tissue to 


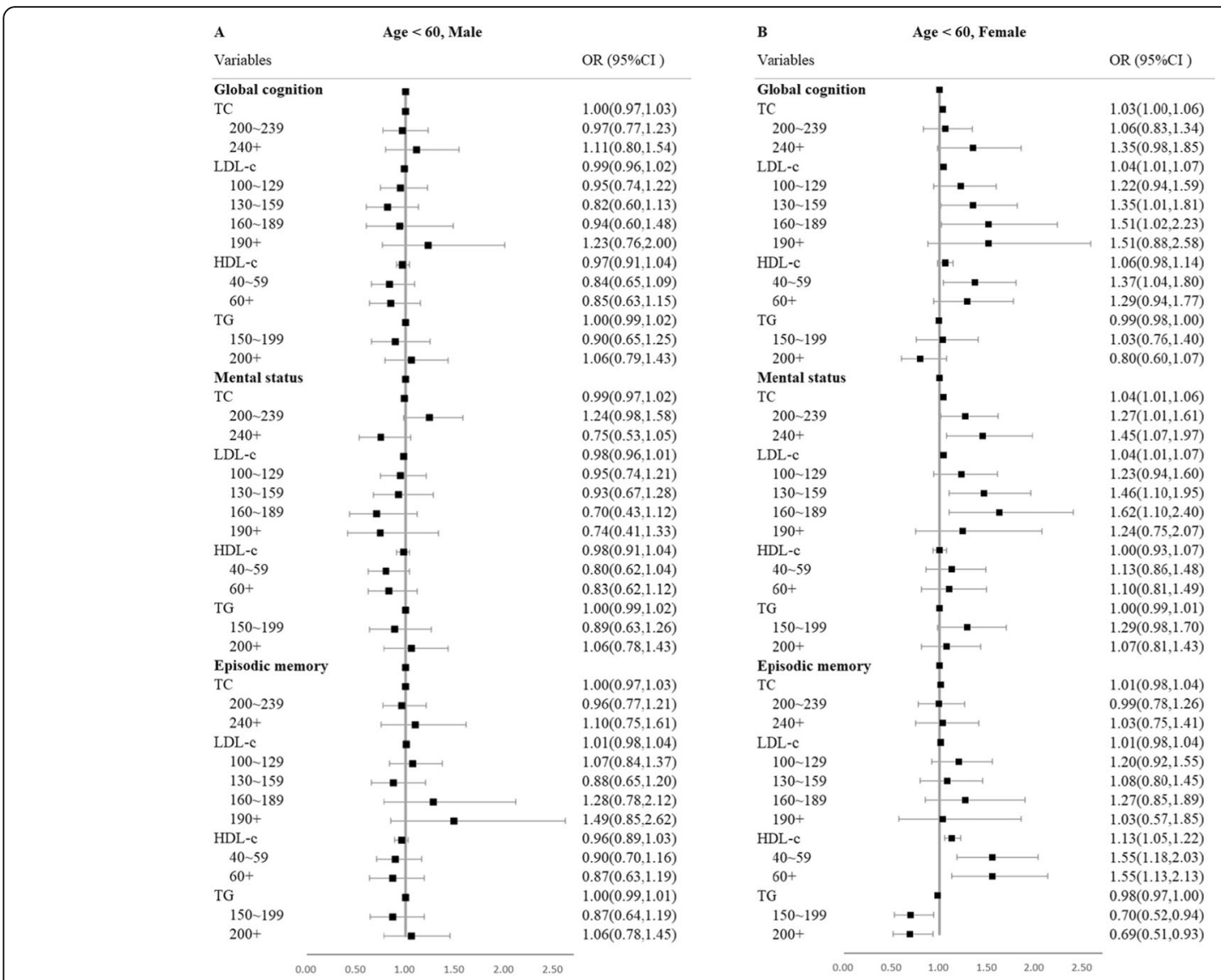

Fig. 1 Forest plot of subgroup analyses for age $<60$, male (a), and age $<60$, female (b), in ordinal logistic regressions. OR, odds ratio; Cl, confidence interval. Adjusting for baseline education, marital status, registered residence, body mass index, alcohol use, smoking status, diabetes, hypertension, social activity, health insurance status, and lipid-lowering medication use

the liver and thus prevent atherosclerosis, which may contribute to maintaining cognition. However, a slightly adverse effect of HDL-C on global cognition in women was also observed, which is consistent with a Chinese cohort study [31]. This novel finding of HDL-C (showing different effects between sexes) was not previously reported. After a systematic literature review, we reasonably hypothesized that this discrepancy might be caused by the different effects of the composition of HDL particles on cognition. HDL particles, a mass of substances in heterogeneous sizes and structures, have various biological functions [53]. Among these particles, small and dense particles were considered to have better atheroprotective properties [53] and reduce the total risk of stroke [54]. In general, men have more small HDL particles compared with women $[55,56]$, thereby yielding a protective impact of HDL-C on cognition in men. However, in women, these particles were associated with a harmful effect in this study, especially in the HDL-C $\geq$ $60 \mathrm{mg} / \mathrm{dl}$ group. As shown in our study, higher TC and LDL-C levels were noted in these subjects, the adverse effect of which might outweigh the weak protective effect of small HDL particles. Women were more likely to have higher TC and LDL-C levels than men with similar HDL-c levels [57], which would further lead to the contrary impact of HDL-C between sexes in this study.

Of note, all the significant associations mentioned above were merely observed for global cognition and mental status. Even though the global cognition outcomes of this study might be driven by mental status outcomes, but separately, there was indeed no effect found in the episodic memory, suggesting that mental status might be more likely to be affected by lipids. In CHARLS, the mental status test provided a measure of attention, numerical ability and time orientation, which was dominated by the frontal areas in the brain [58]. Based on this notion, there was an underlying possibility that changes in lipids were more likely to affect the frontal areas, while this hypothesis should be further tested [22]. However, our results of mental status were in line with those of the Three-City study, which indicated that frontal executive might be more vulnerable in early cognitive impairment [22]. But it should be noted 


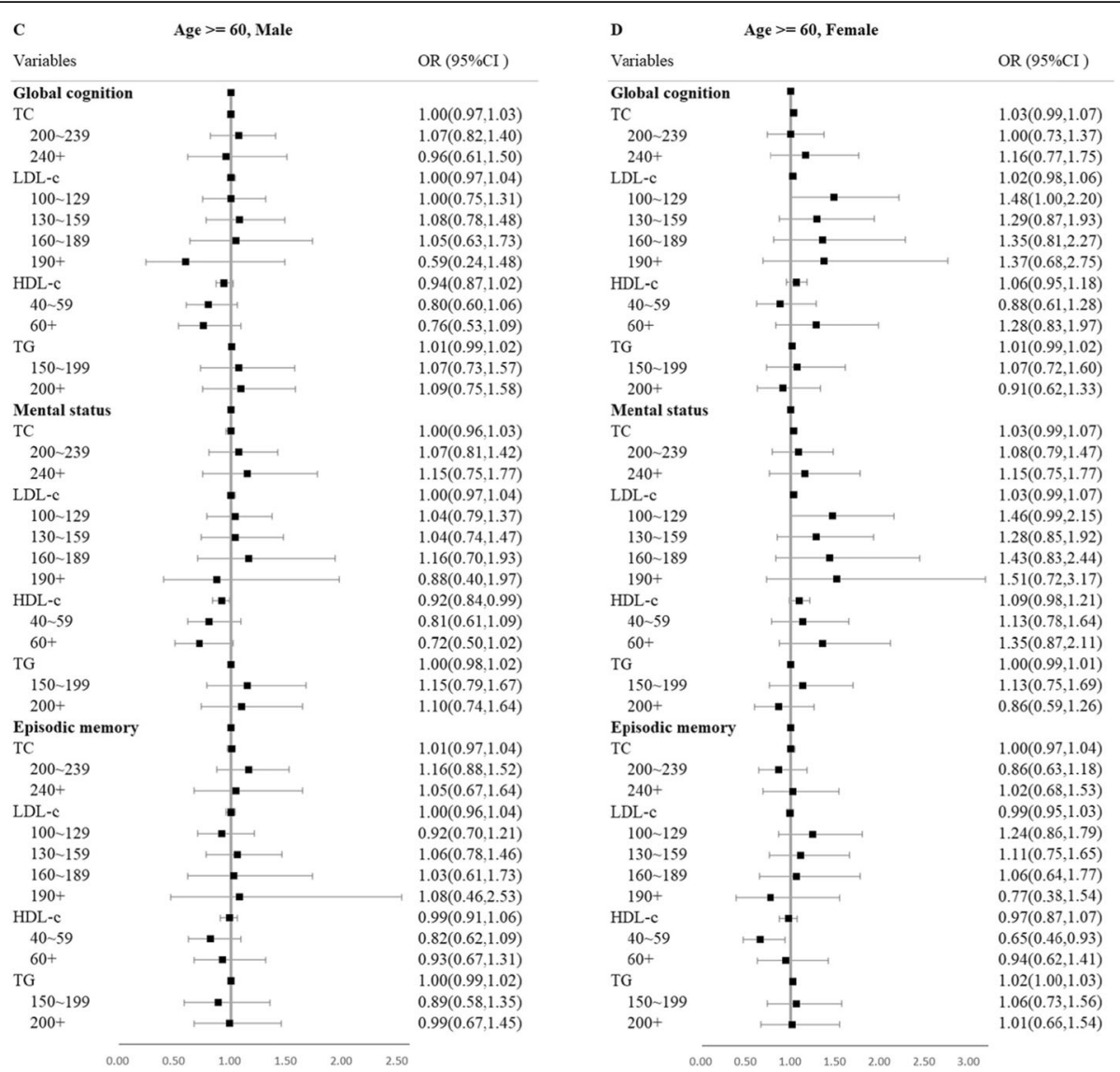

Fig. 2 Forest plot of subgroup analyses for age $\geq 60$, male (c), and age $\geq 60$, female (d), in ordinal logistic regressions. OR, odds ratio; $\mathrm{Cl}$, confidence interval. Adjusting for baseline education, marital status, registered residence, body mass index, alcohol use, smoking status, diabetes, hypertension, social activity, health insurance status, and lipid-lowering medication use

that we also found very few positive signals on episodic memory in our later subgroup analyses.

According to the subgroup analyses of age, the significant influences of lipids on cognition were mainly noted in younger people (<60 years), whereas the effects seemed to disappear in the elderly group ( $\geq 60$ years). Consistent with our finding, a recent meta-analysis including 17 cohort studies with 23,338 participants demonstrated that higher serum cholesterol was associated with greater cognitive decline or any dementia in midlife (40-60 years), but no association was found in late-life (> 60 years) [59]. As individuals aged, the effect of TC on cardiovascular diseases is gradually attenuated [60, 61]. Aging might also lead to several changes in the arterial wall that would later decrease its susceptibility to cholesterol levels in the blood [61]. Additionally, due to the deterioration in brain structure and function, cognitive decline in the elderly mainly occurred naturally with aging, which might take place earlier than the effect of lipids on cognition. Moreover, it also should be noted that the proportion of illiterate females in CHARLS was higher than that of men, which is consistent with the
2010 educational status of China in the middle-aged and elderly [62]. As high education levels have been proposed as a proxy for cognitive reserve and are an important protective factor for cognition [63, 64], this large educational difference might have significant impacts on later cognition between sexes. However, lipid-cognition associations in this study remained after correction for education by multivariate adjustment, indicating the potential effect of lipids on later cognition. Furthermore, subgroup analyses based on education showed that the associations between lipids and cognition varied among different educational groups and mainly occurred in females with high educational level, indicating that specific lipid management is needed for both sexes based on educational background [31].

\section{Limitations}

A few limitations existed in this study. First, the cognitive domains tested in this study were relatively limited. However, to the best of our knowledge, mental status and episodic memory can be used to represent the majority domains of cognitive functions [65]. Second, we 
could not exclude the possibility of residual confounders due to the sex differences on baseline education and other unknown vascular risk factors as well as the absence of information on APOE genotype and dietary cholesterol intake. However, after the multivariable adjustment, the majority of potential confounding effect would be controlled. Third, the lack of imaging diagnoses and molecular markers for neurodegenerative diseases limited our explorations of the mechanism linking high lipid levels and cognitive decline.

\section{Conclusion}

In conclusion, our study suggested that women tended to be more susceptible to adverse effects of higher TC and LDL-C levels on global cognition and mental status. Higher serum HDL-C levels might have beneficial effects on cognition in men but adverse effects in women. Further work is needed to explore the potential mechanisms of the sex-specific associations between lipids and cognitive decline. The results from our study suggested that lipids are a potential treatable factor and maintaining serum cholesterol levels at an appropriate range may have a positive effect on cognitive health.

\section{Supplementary information}

Supplementary information accompanies this paper at https://doi.org/10. 1186/s13195-020-00731-1.

Additional file 1: Supplementary Table 1. TC, LDL-C levels in different HDL-c grades. Supplementary Table 2. Adjusted odds ratio in 5-year cognitive change in different plasma lipid levels across education levels, OR (95\%Cl. Abbreviations: OR, odds ratio; Cl, confidence interval; LDL-C, low-density lipoprotein cholesterol; HDL-c, high-density lipoprotein cholesterol. ${ }^{\dagger}$ Adjusted for baseline age, marital status, registered residence, body mass index, alcohol use, smoking status, diabetes, hypertension, social activity, health insurance status and lipid-lowering medication use. * $P<0.05$ after Bonferroni correction. Supplementary Table 3. Adjusted odds ratio in 5-year cognitive change per $10 \mathrm{mg} / \mathrm{dL}$ lipids at baseline across sensitivity analyses, OR $(95 \% \mathrm{Cl})$. Abbreviations: $\mathrm{OR}$, odds ratio; $\mathrm{Cl}$, confidence interval; LDL-c, low-density lipoprotein cholesterol; HDL-C, high-density lipoprotein cholesterol. ${ }^{\dagger}$ Adjusted for baseline age, education, marital status, registered residence, body mass index, alcohol use, smoking status, diabetes, hypertension, social activity, health insurance status and lipid-lowering medication use. ${ }^{*} P<0.05$, ${ }^{*} P<0.01$.

\section{Abbreviations}

HDL-C: High-density lipoprotein cholesterol; LDL-C: Low-density lipoprotein cholesterol; TC: Total cholesterol; TG: Triglycerides; MST: Mental Status Test; WRT: Word Recall Test

\section{Acknowledgements}

Not applicable.

\section{Authors' contributions}

$L L, C Z, X L, Y S, S W$ and SZ had full access to the aggregate analysis data in the study and take responsibility for the integrity of the data and the accuracy of the data analysis. LL, YS and SW were responsible for the study concept, and $L L, C Z, X L, L X, J F, X L, Y S$ and $S W$ were responsible for the study design. $L L$ and SW were responsible for the statistical analysis. All authors were involved in the acquisition and interpretation of data. $L L$ and $C Z$ drafted the manuscript. All authors critically revised the manuscript for important intellectual content. The authors read and approved the final manuscript.

\section{Funding}

This study was supported by the National Natural Science Foundation (81922016, 81870607) and Shandong Provincial Natural Science Foundation (ZR2019JQ25) of China. The funders/sponsors had no role in the design and conduct of the study; collection, management, analysis, or interpretation of the data; preparation, review, or approval of the manuscript; and decision to submit the manuscript for publication.

\section{Availability of data and materials}

The datasets that support the findings of the current study are available from the corresponding author on reasonable request. The data are publicly available and can be downloaded at http://charls.pku.edu.cn/index/zh-cn.html.

\section{Ethics approval and consent to participate}

The survey and the whole protocol were approved by the Institutional Review Board of Peking University, China (IRB00001052-11015). All subjects provided written informed consent to participate at each study visit.

\section{Consent for publication}

Not applicable.

\section{Competing interests}

The authors report no competing interests.

\section{Author details}

${ }^{1}$ Department of Epidemiology and Biostatistics, School of Public Health, Peking University, 38 Xueyuan Road, Haidian District, Beijing 100191, China. ${ }^{2}$ Department of Endocrinology, Shandong Provincial Hospital Affiliated to Shandong First Medical University, 324 Jing 5 road, Huaiyin District, Jinan 250021, China. ${ }^{3}$ Beijing Dementia Key Laboratory, National Clinical Research Center for Mental Disorders, Peking University Sixth Hospital (Institute of Mental Health), 51 Huayuanbei Road, Haidian District, Beijing 100191, China. ${ }^{4}$ School of Public Health, Peking University, 38 Xueyuan Road, Haidian District, Beijing 100191, China. ${ }^{5}$ Research Center of Clinical Epidemiology, Peking University Third Hospital, 49 Huayuan North Road, Haidian District, Beijing 100191, China. ${ }^{6}$ Center for Intelligent Public Health, Institute for Artificial Intelligence, Peking University, Beijing 100191, China.

Received: 6 July 2020 Accepted: 23 November 2020 Published online: 07 December 2020

\section{References}

1. Roberts R, Knopman DS. Classification and epidemiology of $\mathrm{MCl}$. Clin Geriatr Med. 2013;29:753-72.

2. Llibre Rodriguez JJ, Ferri CP, Acosta D, Guerra M, Huang Y, Jacob KS, et al. Prevalence of dementia in Latin America, India, and China: a populationbased cross-sectional survey. Lancet. 2008;372:464-74.

3. van Oijen M, de Jong FJ, Witteman JC, Hofman A, Koudstaal PJ, Breteler MM. Atherosclerosis and risk for dementia. Ann Neurol. 2007:61:403-10.

4. Gonzalez HM, Tarraf W, Harrison K, Windham BG, Tingle J, Alonso A, et al. Midlife cardiovascular health and 20-year cognitive decline: Atherosclerosis Risk in Communities Study results. Alzheimers Dement. 2018;14:579-89.

5. Ma C, Yin Z, Zhu P, Luo J, Shi X, Gao X. Blood cholesterol in late-life and cognitive decline: a longitudinal study of the Chinese elderly. Mol Neurodegener. 2017;12:24.

6. Power MC, Rawlings A, Sharrett AR, Bandeen-Roche K, Coresh J, Ballantyne CM, et al. Association of midlife lipids with 20-year cognitive change: a cohort study. Alzheimers Dement. 2018;14:167-77.

7. Whitmer RA, Sidney S, Selby J, Johnston SC, Yaffe K. Midlife cardiovascular risk factors and risk of dementia in late life. Neurology. 2005;64:277-81.

8. Schilling S, Tzourio C, Soumare A, Kaffashian S, Dartigues JF, Ancelin ML, et al. Differential associations of plasma lipids with incident dementia and dementia subtypes in the 3C Study: a longitudinal, population-based prospective cohort study. PLoS Med. 2017;14:e1002265.

9. Jick H, Zornberg GL, Jick SS, Seshadri S, Drachman DA. Statins and the risk of dementia. Lancet. 2000;356:1627-31.

10. Ong KL, Morris MJ, McClelland RL, Hughes TM, Maniam J, Fitzpatrick AL, et al. Relationship of lipids and lipid-lowering medications with cognitive 
function: the multi-ethnic study of atherosclerosis. Am J Epidemiol. 2018; 187:767-76.

11. Li G, Shofer JB, Kukull WA, Peskind ER, Tsuang DW, Breitner JC, et al. Serum cholesterol and risk of Alzheimer disease: a community-based cohort study. Neurology. 2005;65:1045-50.

12. Reitz C, Luchsinger J, Tang MX, Manly J, Mayeux R. Impact of plasma lipids and time on memory performance in healthy elderly without dementia. Neurology. 2005;64:1378-83.

13. van den Kommer TN, Dik MG, Comijs HC, Fassbender K, Lutjohann D, Jonker C. Total cholesterol and oxysterols: early markers for cognitive decline in elderly? Neurobiol Aging. 2009:30:534-45.

14. Benito-Leon J, Vega-Quiroga S, Villarejo-Galende A, Bermejo-Pareja F. Hypercholesterolemia in elders is associated with slower cognitive decline: a prospective, population-based study (NEDICES). J Neurol Sci. 2015;350:69-74.

15. Elias PK, Elias MF, D'Agostino RB, Sullivan LM, Wolf PA. Serum cholestero and cognitive performance in the Framingham Heart Study. Psychosom Med. 2005;67:24-30.

16. Kolovou GD, Anagnostopoulou KK, Damaskos DS, Bilianou HI, Mihas C, Milionis $\mathrm{HJ}$, et al. Gender differences in the lipid profile of dyslipidemic subjects. Eur J Intern Med. 2009;20:145-51.

17. Li R, Singh M. Sex differences in cognitive impairment and Alzheimer's disease. Front Neuroendocrinol. 2014;35:385-403.

18. Mielke MM, Ferretti MT, Iulita MF, Hayden K, Khachaturian AS. Sex and gender in Alzheimer's disease - does it matter? Alzheimers Dement. 2018;14: $1101-3$.

19. Shaw LJ, Bugiardini R, Merz CNB. Women and ischemic heart disease. J Am Coll Cardiol. 2009:54:1561-75.

20. Jia L, Quan M, Fu Y, Zhao T, Li Y, Wei C, et al. Dementia in China: epidemiology, clinical management, and research advances. Lancet Neurol. 2020;19:81-92

21. Reynolds CA, Gatz M, Prince JA, Berg S, Pedersen NL. Serum lipid levels and cognitive change in late life. J Am Geriatr Soc. 2010;58:501-9.

22. Ancelin ML, Ripoche E, Dupuy AM, Samieri C, Rouaud O, Berr C, et al. Gender-specific associations between lipids and cognitive decline in the elderly. Eur Neuropsychopharmacol. 2014;24:1056-66

23. Zhao Y, Hu Y, Smith JP, Strauss J, Yang G. Cohort profile: the China Health and Retirement Longitudinal Study (CHARLS). Int J Epidemiol. 2014;43:61-8.

24. Sha T, Cheng W, Yan Y. Prospective associations between pulse pressure and cognitive performance in Chinese middle-aged and older population across a 5-year study period. Alzheimers Res Ther. 2018:10:29.

25. Lu Y, Wang P, Zhou T, Lu J, Spatz ES, Nasir K, et al. Comparison of prevalence, awareness, treatment, and control of cardiovascular risk factors in China and the United States. J Am Heart Assoc. 2018;7:16. https://doi.org/ 10.1161/JAHA.117.007462.

26. Lei X, Hu Y, McArdle JJ, Smith JP, Zhao Y. Gender differences in cognition among older adults in China. J Hum Resour. 2012;47:951-71.

27. Yang $\mathrm{X}$, Pan A, Gong J, Wen $\mathrm{Y}, \mathrm{Ye} \mathrm{Y}, \mathrm{Wu} \mathrm{JH}$, et al. Prospective associations between depressive symptoms and cognitive functions in middle-aged and elderly Chinese adults. J Affect Disord. 2020;263:692-7.

28. Baars MAE, van Boxtel MPJ, Dijkstra JB, Visser PJ, van den Akker M, Verhey FRJ, et al. Predictive value of mild cognitive impairment for dementia. The influence of case definition and age. Dement Geriatr Cogn Disord. 2009;27: $173-81$

29. Seo EH, Lee DY, Kim SG, Kim KW, Kim DH, Kim BJ, et al. Validity of the telephone interview for cognitive status (TICS) and modified TICS (TICSm) for mild cognitive imparment (MCl) and dementia screening. Arch Gerontol Geriatr. 2011;52:e26-30

30. Mielke MM, Zandi PP, Shao H, Waern M, Ostling S, Guo X, et al. The 32-year relationship between cholesterol and dementia from midlife to late life. Neurology. 2010;75:1888-95.

31. An $Y$, Zhang $X$, Wang $Y$, Wang $Y$, Liu W, Wang $T$, et al. Longitudinal and nonlinear relations of dietary and serum cholesterol in midlife with cognitive decline: results from EMCOA study. Mol Neurodegener. 2019;14:51.

32. Zhang L, Yang J, Liao Z, Zhao X, Hu X, Zhu W, et al. Association between diabetes and cognitive function among people over 45 years old in China: a cross-sectional study. Int J Environ Res Public Health. 2019;16:12. https:// doi.org/10.3390/ijerph16071294

33. Adult Treatment Panel III. Executive summary of the third report of the National Cholesterol Education Program (NCEP) Expert Panel on Detection, Evaluation, And Treatment of High Blood Cholesterol In Adults (Adult Treatment Panel III). JAMA. 2001;285:2486-97.
34. Steinberg HO, Bayazeed B, Hook G, Johnson A, Cronin J, Baron AD. Endothelial dysfunction is associated with cholesterol levels in the high normal range in humans. Circulation. 1997;96:3287-93.

35. Craik FIM, Rose NS. Memory encoding and aging: a neurocognitive perspective. Neurosci Biobehav Rev. 2012;36:1729-39.

36. Kim J-Y, Park S-J, Kim S-K, Kim C-S, Kim T-H, Min S-H, et al. Pesticide exposure and cognitive decline in a rural South Korean population. PLoS One. 2019;14:e0213738.

37. Cedres N, Machado A, Molina Y, Diaz-Galvan P, Hernández-Cabrera JA, Barroso J, et al. Subjective cognitive decline below and above the age of 60: a multivariate study on neuroimaging, cognitive, clinical, and demographic measures. J Alzheimers Dis. 2019;68:295-309.

38. Yaffe K, Barrett-Connor E, Lin F, Grady D. Serum lipoprotein levels, statin use, and cognitive function in older women. Arch Neurol. 2002;59:378-84.

39. Deckers K, Schievink SHJ, Rodriquez MMF, van Oostenbrugge RJ, van Boxtel MPJ, Verhey FRJ, et al. Coronary heart disease and risk for cognitive impairment or dementia: systematic review and meta-analysis. PLoS One. 2017;12:e0184244

40. van Vliet $P$, van de Water W, de Craen AJM, Westendorp RGJ. The influence of age on the association between cholesterol and cognitive function. Exp Gerontol. 2009:44:112-22.

41. Refolo LM, Malester B, LaFrancois J, Bryant-Thomas T, Wang R, Tint GS, et al. Hypercholesterolemia accelerates the Alzheimer's amyloid pathology in a transgenic mouse model. Neurobiol Dis. 2000;7:321-31.

42. Thirumangalakudi L, Prakasam A, Zhang R, Bimonte-Nelson H, Sambamurti $\mathrm{K}$, Kindy MS, et al. High cholesterol-induced neuroinflammation and amyloid precursor protein processing correlate with loss of working memory in mice. J Neurochem. 2008;106:475-85.

43. Mielke MM, Xue QL, Zhou J, Chaves PH, Fried LP, Carlson MC. Baseline serum cholesterol is selectively associated with motor speed and not rates of cognitive decline: the Women's Health and Aging Study II. J Gerontol A Biol Sci Med Sci. 2008:63:619-24.

44. Zhao LP, Kolonel LN. Efficiency loss from categorizing quantitative exposures into qualitative exposures in case-control studies. Am J Epidemiol. 1992;136:464-74.

45. Laughlin GA, McEvoy LK, von Muhlen D, Daniels LB, Kritz-Silverstein D, Bergstrom J, et al. Sex differences in the association of Framingham Cardiac Risk Score with cognitive decline in community-dwelling elders without clinical heart disease. Psychosom Med. 2011;73:683-9.

46. Sachdev PS, Parslow R, Wen W, Anstey K, Easteal S. Sex differences in the causes and consequences of white matter hyperintensities. Neurobiol Aging. 2009;30:946-56

47. Vegeto E, Villa A, Della Torre S, Crippa V, Rusmini P, Cristofani R, et al. The role of sex and sex hormones in neurodegenerative diseases. Endocr Rev. 2020:41:128. https://doi.org/10.1210/endrev/bnz005.

48. Mielke MM, Vemuri P, Rocca WA. Clinical epidemiology of Alzheimer's disease: assessing sex and gender differences. Clin Epidemiol. 2014;6: 37-48.

49. Giedd JN, Raznahan A, Mills KL, Lenroot RK. Review: magnetic resonance imaging of male/female differences in human adolescent brain anatomy. Biol Sex Differ. 2012;3:19

50. Katzman R, Terry R, DeTeresa R, Brown T, Davies P, Fuld P, et al. Clinical, pathological, and neurochemical changes in dementia: a subgroup with preserved mental status and numerous neocortical plaques. Ann Neurol. 1988;23:138-44

51. Sundermann EE, Maki PM, Rubin LH, Lipton RB, Landau S, Biegon A. Female advantage in verbal memory: evidence of sex-specific cognitive reserve. Neurology. 2016;87:1916-24.

52. Svensson T, Sawada N, Mimura M, Nozaki S, Shikimoto R, Tsugane S. The association between midlife serum high-density lipoprotein and mild cognitive impairment and dementia after 19 years of follow-up. Trans Psychiatry. 2019;9:26

53. Camont L, Chapman MJ, Kontush A. Biological activities of HDL subpopulations and their relevance to cardiovascular disease. Trends Mol Med. 2011:17:594-603.

54. Chei CL, Yamagishi K, Kitamura A, Kiyama M, Imano H, Ohira T, et al. Highdensity lipoprotein subclasses and risk of stroke and its subtypes in Japanese population: the Circulatory Risk in Communities Study. Stroke. 2013:44:327-33

55. Freedman DS, Otvos JD, Jeyarajah EJ, Shalaurova I, Cupples LA, Parise $H$, et al. Sex and age differences in lipoprotein subclasses measured by nuclear 
magnetic resonance spectroscopy: the Framingham Study. Clin Chem. 2004; 50:1189-200.

56. Swiger KJ, Martin SS, Blaha MJ, Toth PP, Nasir K, Michos ED, et al. Narrowing sex differences in lipoprotein cholesterol subclasses following mid-life: the very large database of lipids (VLDL-10B). J Am Heart Assoc. 2014;3:e000851.

57. Li S, Zhang L, Wang X, Chen Z, Dong Y, Zheng C, et al. Status of dyslipidemia among adults aged 35 years and above in China. Chin Circ J. 2019:34:681-7.

58. Roca M, Parr A, Thompson R, Woolgar A, Torralva T, Antoun N, et al. Executive function and fluid intelligence after frontal lobe lesions. Brain. 2010;133:234-47.

59. Anstey K, Ashby-Mitchell K, Peters R. Updating the evidence on the association between serum cholesterol and risk of late-life dementia: review and meta-analysis. J Alzheimers Dis. 2017;56:215-28.

60. Simons LA, Simons J, Friedlander Y, McCallum J. Cholesterol and other lipids predict coronary heart disease and ischaemic stroke in the elderly, but only in those below 70 years. Atherosclerosis. 2001;159:201-8.

61. Krumholz HM, Seeman TE, Merrill SS, Mendes de Leon CF, Vaccarino V, Silverman DI, et al. Lack of association between cholesterol and coronary heart disease mortality and morbidity and all-cause mortality in persons older than 70 years. JAMA. 1994;272:1335-40.

62. Zhang $\mathrm{H}$. The current situation and changes of the education level in the elderly of China. Chin J Gerontol. 2016;36:e1215-6.

63. Stern Y. Cognitive reserve in ageing and Alzheimer's disease. Lancet Neurol. 2012;11:1006-12.

64. Stern Y. Cognitive reserve. Neuropsychologia. 2009:47:2015-28.

65. Qin T, Yan M, Fu Z, Song Y, Lu W, Fu A, et al. Association between anemia and cognitive decline among Chinese middle-aged and elderly: evidence from the China health and retirement longitudinal study. BMC Geriatr. 2019; 19:305.

\section{Publisher's Note}

Springer Nature remains neutral with regard to jurisdictional claims in published maps and institutional affiliations.

Ready to submit your research? Choose BMC and benefit from:

- fast, convenient online submission

- thorough peer review by experienced researchers in your field

- rapid publication on acceptance

- support for research data, including large and complex data types

- gold Open Access which fosters wider collaboration and increased citations

- maximum visibility for your research: over $100 \mathrm{M}$ website views per year

At $\mathrm{BMC}$, research is always in progress.

Learn more biomedcentral.com/submissions 